\title{
PHYSIOLOGICAL ONTOGENY.
}

\section{A. Chicken Embryos.}

\section{On the Rate of the Heart Beat during the Development of Chicken Embryos.}

BY ALFRED E. COHN, M.D.

With the Assistance of Edith L. Wile.*

(From the Hospital of The Rockefeller Institute for Medical Research.)

(Received for publication, March 2, 1925.)

A function so intimately associated with the developing life of the organism as is the rate of the heart beat should be studied exactly. It may have importance from the point of view of the development of the whole organism and its metabolism, of the circulation, and of the differentiation of the function of stimulus production on the part of the various structures which ultimately form the adult heart.

A systematic study of the pulse rate throughout the embryonic cycle of the life of the chicken does not seem before to have been undertaken. Wernicke (1) made two series of partial observations for this purpose, one between the 46th hour and 7 th day in which the attempt was made to maintain the temperature constant by means of a sand bath, and a second between 67 hours and 11 days in which the shell and outer shell membrane were removed and the whole egg, embryo and yolk, placed in a water bath. In both series large numbers of experiments were performed at each age investigated, but when the data are plotted a consistent rate curve cannot be drawn through the points, even when cases of like temperature are compared. Why order cannot be made from these experiments it is difficult, perhaps impossible, to understand.

In the observations reported it was our purpose to carry on the study in such a manner that, if there were an underlying order in the rates which the heart develops at succeeding stages throughout its embryonic life, that fact would emerge from the information collected.

* We are indebted to Miss Alma Rosenthal for aid in the early part of these experiments. 
It seemed to be important to take care not only of the conditions within the incubator in which the eggs developed but more important still of the room in which the observations were made. After futile attempts had been made to work in an ordinary room at ordinary room temperature, a room already described (2) was built in which a constant temperature $\left(38^{\circ} \mathrm{C} . \pm 1.0^{\circ}\right)$ could be maintained. The eggs were of white leghorn hens, obtained from the source already indicated. They were incubated under standard conditions (2). In order to count the rate of the heart a small window was cut or picked out of the shell, care having been taken, by maintaining the position constant during the preceding 12 hours and by candling, to ascertain the position of the embryo. Counting was facilitated by observing the heart itself in the younger embryos through a binocular dissecting microscope. The field was illuminated by focusing a beam of light on the window and the tissues within. In order to eliminate the influence of heat rays a water bath through which a stream of cold running water was directed was interposed in the course of the light beam. In the older embryos when the heart was no longer visible the count was obtained either by observing the pulsation of a vessel, or of the stream within the vessel, or sometimes the motions of a beam of light to which the pulsations of the heart or of a vessel were communicated. Counts of half a minute's duration each were made for about 5 minutes.

To estimate the stage of development at which the embryo had arrived we used four means. (1) We knew the incubation time; (2) we weighed the embryos after the counts were made; (3) we made drawings of the area of the heart and measured these; and (4) we compared the morphology of the heart with a series of drawings of hearts of known age at successive periods. The drawings for this last estimation were made in our laboratory during the course of this and of other work and they were checked against the data found in the literature. As will be seen later, the variation in the heart rate at any given stage was so great that the use of it in an attempt tobe precise in respect to the state of development was abandoned. In the end weighing proved the best control. If the average weight at each age of the embryos in this series is compared with Murray's (3) curve showing the relation of weight to age a close correspondence between our findings and his is evident (Fig. 1).

The age can apparently be established also by measuring the area of the heart. The hearts were excised, after the embryos had been weighed, and were floated, to avoid anteroposterior flattening, in chicken serum contained in a receptacle formed by a glass ring secured to a glass slide by paraffin. The outline of the 
heart was drawn by means of an Abbe drawing apparatus. Directly afterward, a stage micrometer was placed on the microscope stage at the level where the heart had been and its scale was drawn on the same paper as the outline of the heart. The magnification of the area of the heart was later computed from the drawing. If the logarithms of the areas $(\times 10)$ so found are plotted against the

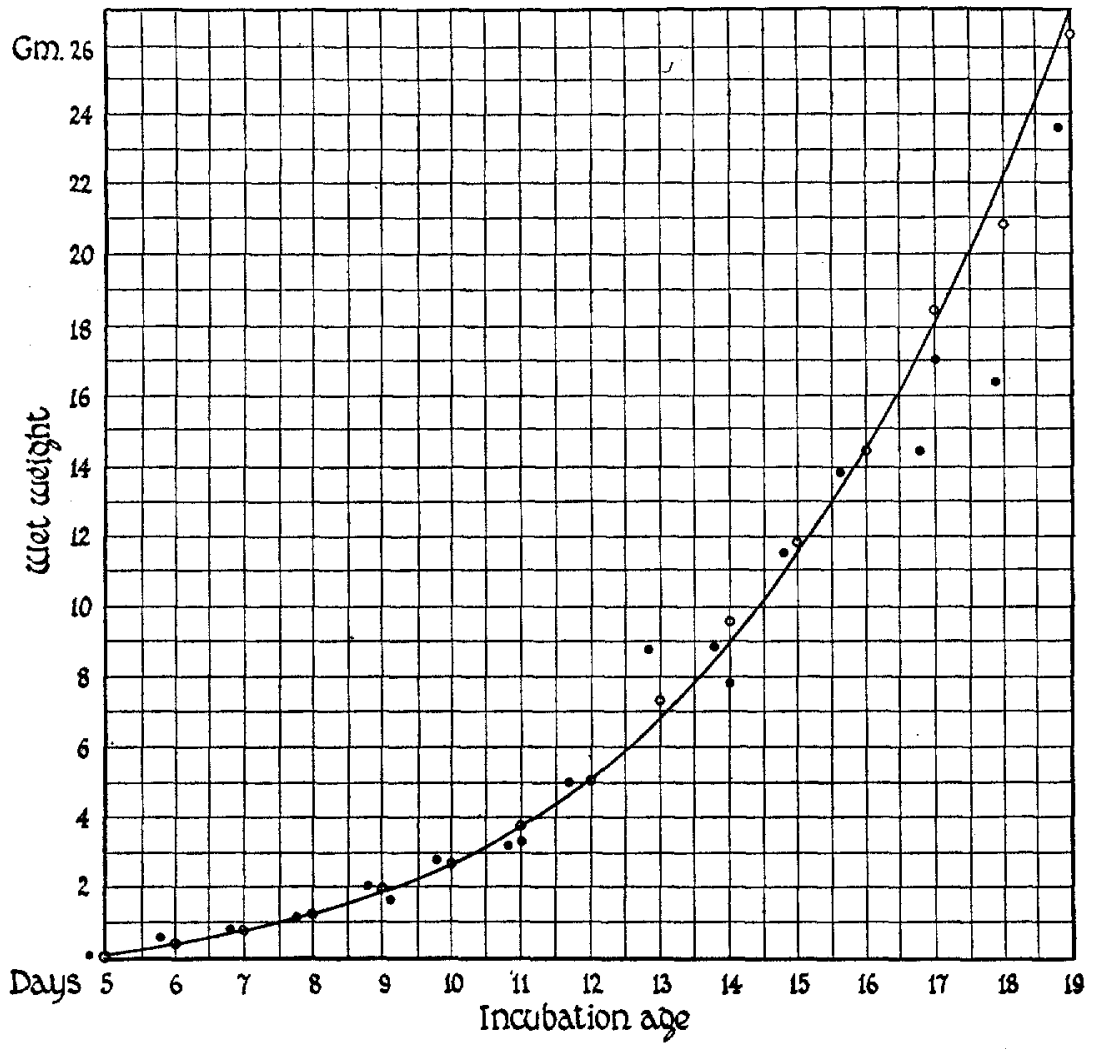

FIG. 1. The curve and the white circles (0) shown are the same as those of Fig. 1 in Paper III of this series (3). The solid black circles () are the average weights at the ages indicated of the embryos studied in this series.

age a smooth curve (Fig. 2) can be drawn through points. It is apparent that the greatest rate of increase in the area of the heart, as in the weight of the embryo, is found in the first 4 days. It is clear, then, that the age of embryos can be known in terms either of the weight of the embryo, as Murray has shown, or of the area of the heart, and that these measurements are apparently functions of the incubation time.

No more than a qualitative statement can be made on the relation of the 
morphology of the heart to age. Before the 7 th day the estimate that was made of the probable age of the embryos based on comparison with the drawings corresponded closely with the age as ascertained by the other means that were employed. After this time, the changes in form are not sufficiently sharp within brief periods of time to permit judgments of even approximate value to be made.

It proved possible in the manner just described to establish a parallel between the weight, cardiac area, and incubation age of the embryos and throughout the work this was carefully done. The

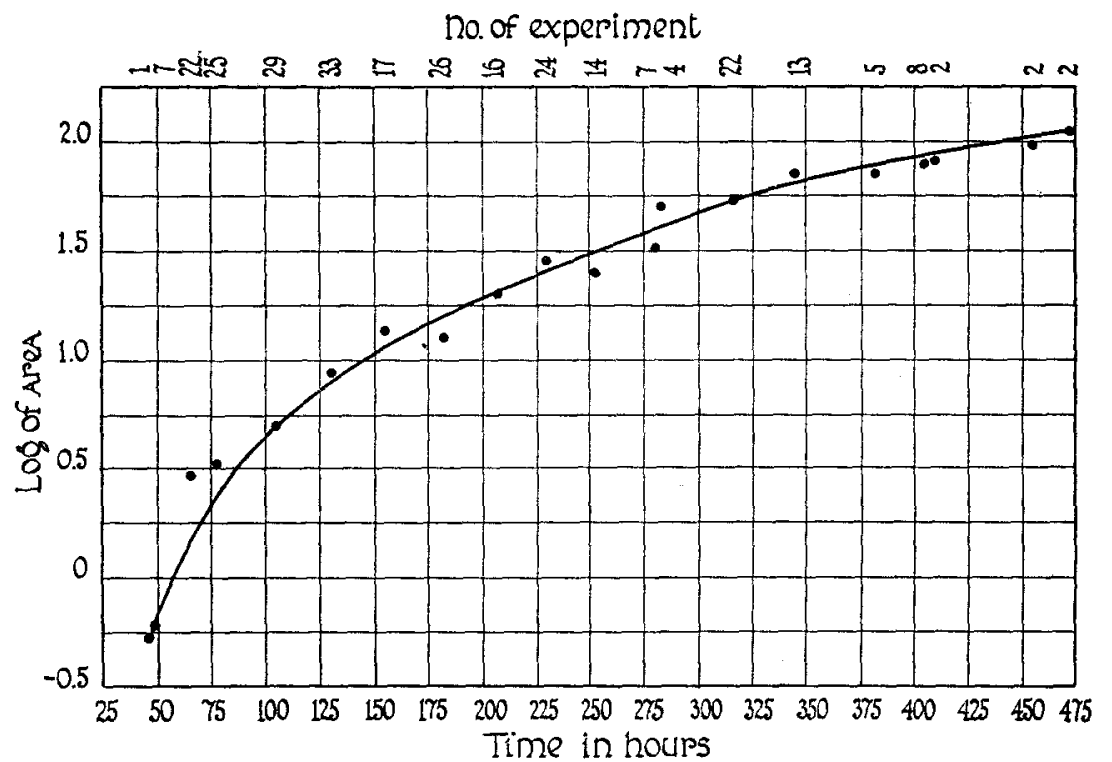

FIG. 2. This curve shows the increase of the projected area of the hearts with time. The method by which the measurements were made is described in the text.

logarithms of the heart rates have been plotted against the time of incubation (Fig. 3). The curve which it has been possible to draw begins at 50 hours ( 2 days, 2 hours) and extends to 450 hours (18 days, 18 hours). Its lowest point is at the beginning. It then rises sharply to about 140 hours ( 5 days, 20 hours) and continues to rise until the period btween the 15 th and the 16th day; that is to say, to 251 beats per minute $(\log =2.40)$. In the last 2 or 3 days of incubation the rate falls slightly (from 251 to 222). 


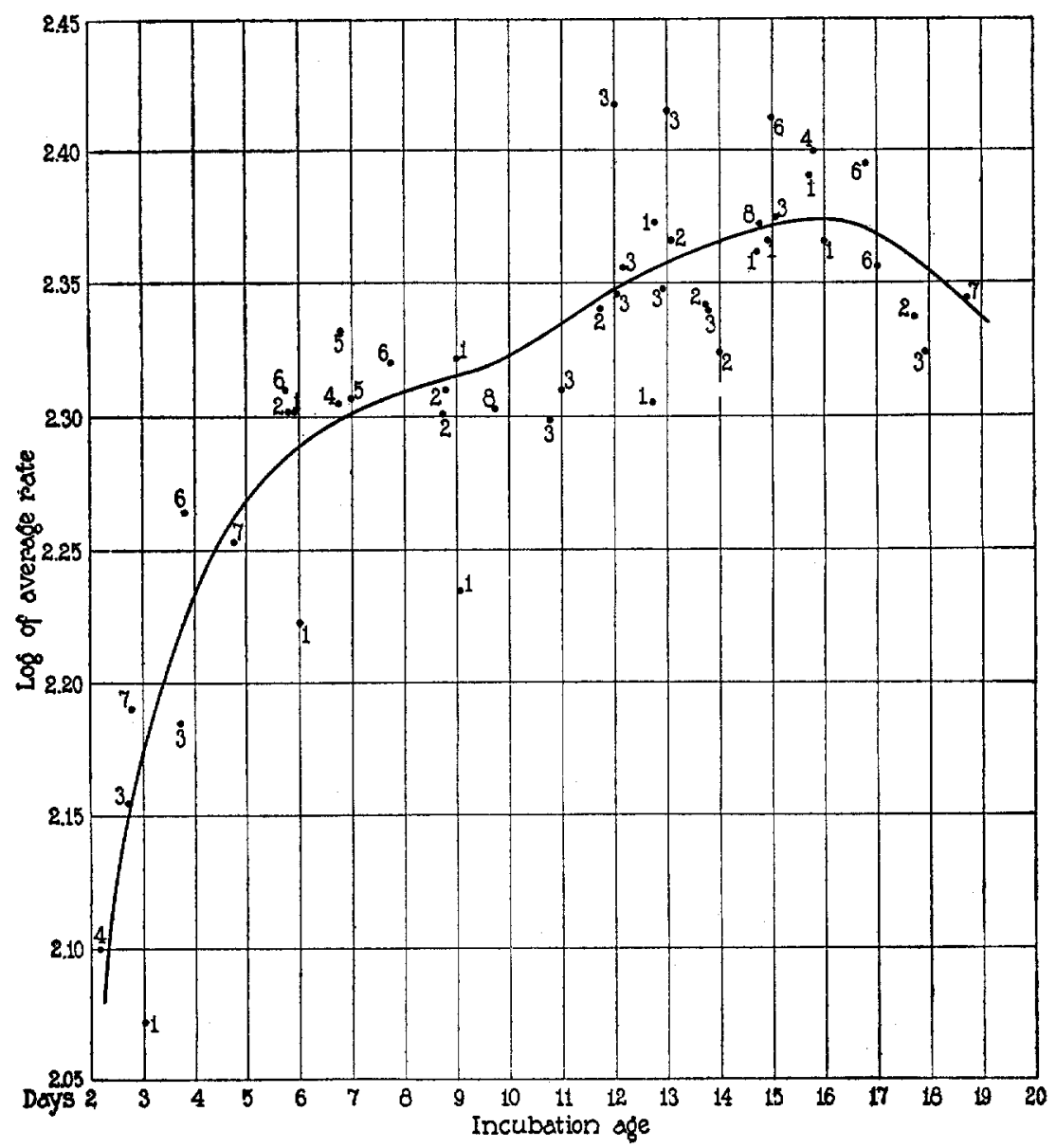

Fig. 3. A curve is shown which serves to indicate that in the course of incubation changes in the rate of the heart beat take place which appear to be of an orderly nature although the deviations from the average are large. The numbers accompanying the points indicate the number of experiments.

DISCUSSION AND SUMMARY.

These experiments show, then, that there are changes in rate which take place during the incubation period and that the direction of the curve expressing them changes in an orderly fashion. No attempt has yet been made to ascertain the influences operative in bringing 
about these alterations. It is our intention, however, to attempt to correlate them with such processes as changes in the rate of the general metabolism of the body, changes in structure of the heart muscle, and if possible with changes in the inherent dynamics of the muscle.

It will be seen that the number of experiments at a given age is relatively speaking small. The temperature in spite of the precaution mentioned has likewise not been uniform, the range in the greatest number (38) of experiments having been $38^{\circ} \mathrm{C} . \pm 1^{\circ}$. A few (four) counts have been made when the temperature was as low as $33.6^{\circ} \mathrm{C}$. and a few (two) also at a temperature as high as $40^{\circ} \mathrm{C}$.

Although a curve as smooth as the one which has been drawn in all probability represents the general course of heart rate change, an explanation of the deviations which have been found is desirable. At least two influences which may be operative suggest themselves. First may be considered the effect of the unavoidable injury incident to cutting a window in the shell and removing so much of the shell membrane as to make the object visible. It has been a uniform practise to discard those eggs in which hemorrhage took place, so that if opening the egg has a share in the difficulty, this operation may be thought of as exerting a mechanical stimulus on a nervous or other controlling mechanism. But whether, especially at the early ages, a mechanism of such a nature exists within the embryo, we do not know. The search for one lies for the present beyond the range of our interest.

A second influence responsible for the relatively large deviation in rate from the average may be that of fluctuations in temperature. The air of the room in which the observations were made was, in a given situation, fairly uniform at $38^{\circ} \mathrm{C} . \pm 1.0^{\circ}$. as the temperature records show. In the earlier experiments the temperature was read at a distance about 1 foot away from the egg. Between this point and the egg a difference unknown to us may well have existed. It is improbable, however, that it can have been more than $2^{\circ} \mathrm{C}$., that is to say, $38^{\circ} \pm 2^{\circ}$. Roughly if the change in rate is approximately 15 beats per degree a maximum deviation of 30 beats may be expected. In point of fact a deviation as great as this did not take place. In later experiments the temperature was read in the immediate vicinity of the eggs, so that a correction can be made more accurately. 
There is in all probability a high degree of uniformity in the rate of the heart of the chicken embryo at each stage of development, although variation is also found. What the extent of the deviation is that may properly be encountered we do not yet know. It is desirable to pursue this subject further. Observations will accordingly be made and will be reported later.

\section{CONCLUSION.}

The study here reported has made possible the presentation of a curve which shows distinctly that changes take place in the rate of the heart beat during the development of the chick embryo.

\section{BIBLIOGRAPHY.}

1. Wernicke, R., Zur Physiologie des embryonalen Herzens, in Samml. physiol. Abhandl., Jena, 1876, series 1, No. 5.

2. Murray, H. A., Jr., J. Gen. Physiol., 1925-26, ix, 1.

3. Murray, H. A., Jr., J. Gen. Physiol., 1925-26, ix, 39. 